Prenat Diagn 2006; 26: 711-718.

DOI: $10.1002 / p d .1486$

\title{
Multi-centre first-trimester screening for Down syndrome in the Netherlands in routine clinical practice
}

\author{
P. C. J. I. Schielen*, M. van Leeuwen-Spruijt, I. Belmouden, L. H. Elvers, M. Jonker \\ and J. G. Loeber
}

Diagnostic Laboratory for Infectious Diseases and Perinatal Screening, National Institute for Public Health and the Environment

(RIVM), Bilthoven, The Netherlands

*Correspondence to: P. C. J. I. Schielen, National Institute for Public Health and the Environment (RIVM), PO Box 1, 3720 BA Bilthoven, The Netherlands. E-mail:

Peter.Schielen@rivm.nl

Objectives This is the first report on the results of a first-trimester combined-test screening programme in the Netherlands in a multi-centre routine clinical setting.

Methods Between July 2002 and May 2004, blood samples were taken from subjects in 44 centres in the Netherlands and sent to our laboratory to assay for maternal serum concentrations of $\mathrm{f} \beta$-hCG and PAPP-A. Fetal nuchal translucency (NT) was measured in the participating centres at a gestational age (GA) of 10-14 weeks. Results of those pregnancies for which a combined biochemical and NT risk was calculated were included in the epidemiological analysis of this study.

Results A total of 4033 singleton pregnancies were included in the analysis. The median maternal age of the analysed group was 36.5 years. The distribution of GA was biphasic, with median GA of 10.3 and 12.1 weeks, respectively. The detection rate using the combined ultrasound and serum screening at a cut-off level of 1 in 250 was $71 \%(15 / 21)$, with a screen-positive rate of $4.7 \%$.

Conclusion The results of this study show that the first-trimester combined test is suitable as a prenatal screening test in a multi-centre routine clinical setting in the Netherlands.

Strict performance evaluation should identify weaknesses in the organisation that impair the performance of the test. Here, the performance of NT was especially identified as a candidate for improvement.

Copyright (c) 2006 John Wiley \& Sons, Ltd.

\section{INTRODUCTION}

Until recently, there has been political uncertainty about the organisation of a national screening programme for Down syndrome. The official policy was to offer women who had reached the age of 36 and were in the $18^{\text {th }}$ week of pregnancy a diagnostic test for Down syndrome. To offer a risk estimation test or to provide general information about these tests was allowed only at the explicit request of the pregnant women. In this scenario of political uncertainty, the Dutch Health Care Inspectorate allowed the Dutch National Institute for Public Health and the Environment (RIVM) to execute an informal screening programme for the triple test in order to centralise the expertise. Thus, since 1990, these tests are almost exclusively performed in the Netherlands by the RIVM. As a consequence of the Dutch 
Prenat Diagn 2006; 26: 711-718.

governmental policy, mainly older women, typically above 36, participated in this informal screening programme. It is of interest that a formal national screening programme for Down syndrome is anticipated to start in early 2007.

There are several prenatal Down syndrome screening strategies, both in the first trimester and second trimester, or combined in both trimesters, all with their specific performance characteristics (Cuckle and Arbuzova, 2004). One of the most commonly used strategies is the first-trimester combined test. Several studies have provided the notion that the firsttrimester combined test has advantages compared to the triple test (Royston and Thompson, 1992; Wald and Hackshaw, 1997; Spencer et al., 1999). The first-trimester combined test is composed of three elements: (1) assay of the serum concentrations of pregnancy-associated plasma protein A (PAPP-A) and the free $B$ subunit of human chorion gonadotrophin ( $\mathrm{f} \beta-\mathrm{hCG}$ ) between 8-14 weeks of the pregnancy, $(2)$ ultrasound measurement of the nuchal translucency (NT subcutaneous oedema in the fetal neck, to be measured at a gestational age (GA) between 10-11 and 14 weeks), and (3) maternal age (Spencer et al., 1999, 2003; Wapner et al., 2003). NT measurement and biochemical assay of PAPP-A and $\mathrm{f} \beta$-hCG can be done simultaneously or consecutively; both scenarios have their advantages and disadvantages (Cuckle and Arbuzova, 2004).

In 2001, the Dutch Health Council stated in an advice to the Dutch Ministry of Health, Welfare and Sports that the introduction of prenatal screening for Down syndrome must not be delayed any longer. As per their opinion, these tests were a better alternative than the prevailing age-based screening (Health Council of the Netherlands, 2001). After determining the statistically modelled median values of the two serum markers, the first-trimester combined-test screening was started at the RIVM in the second half of 2002, again on the request of the Health Care Inspectorate. This screening programme eventually developed into an informal organisation with 44 participating centres (hospitals, centres for prenatal diagnostics and practices for obstetrics, Schielen et al., 2005). Regionally, the centres originate from all parts of the Netherlands, except from the northern regions of the country, and have their own screening programme. The Dutch setting for the first-trimester combined test has more or less developed spontaneously out of the triple test programme.

In the 1990s, measurement of fetal nuchal translucency was introduced in the Netherlands. In the absence of governmental guidance, this grew into an independent and solitary programme of unknown extent, mainly performed in hospitals. While data on the performance of the first-trimester combined test in the setting of a clinical trial are relatively abundant, data on the performance of this test in a multi-centre routine clinical setting are published only occasionally (Stenhouse et al., 2004). This study reports the first results of a routine multicentre Down syndrome screening programme in the Netherlands, which combines fetal NT with biochemistry and maternal age, with special reference to the strengths and weaknesses of the above setting.

\section{PATIENTS AND METHODS}

\section{Subjects, serum markers, gestational age, study design}

This was a multi-centre study for the screening of Down syndrome, carried out between July 2002 and May 2004. Requests were accepted at a GA of 8.0 to 13.6 weeks. Venous blood samples were taken, from which serum was prepared and sent to the laboratory of the RIVM by conventional mail, and stored upon arrival at $4{ }^{\circ} \mathrm{C}$ until analysis. Fetal nuchal translucency of the subjects was measured in the participating centres between 10-14 weeks. The samples were accompanied by a form to record information on maternal age and GA, (e.g. dating scan details and first day of last menstrual period (LMP)), maternal weight, 
Prenat Diagn 2006; 26: 711-718.

insulin-dependent diabetes mellitus (IDDM) status, number of living foetuses and, if known, the NT and the crown-rump length (CRL). To calculate the multiple of the median (MoM) values of the biochemical parameters, the GA at blood sampling as stated on the form by the requestor (gynaecologist or midwife) was used. In $99 \%$ of cases, the GA was stated by the requestor and in $1 \%$ it was calculated at the laboratory of the RIVM (preferably by using dating scan details, otherwise by using the LMP). Analysis of the sera was done with commercially available kits and the AutoDELFIA analyzer (PerkinElmer, Wallac, Turku, Finland). After assay, the samples were stored at $-20 \circ \mathrm{C}$.

Results of reported Down syndrome pregnancies were confirmed by repeat analysis. The request procedure of the first-trimester test at the RIVM demanded that a biochemical analysis was always combined with an NT measurement by either of two scenarios. In the first scenario, Down syndrome risks were calculated at the centres, using their own risk calculation software (Fetal Medicine Foundation (FMF)-derived, or with a table stating the likelihood ratio for a given combination of CRL and NT). In that case, the serum samples were sent to us and these centres were only given the concentrations and MoM values of PAPP-A and $\mathrm{f} \beta$-hCG and the risk based on the biochemical parameters. The combined risks were not reported back to our laboratory. For these tests, a non-combined risk was registered in our database and they were not included in the epidemiological analysis. In the second scenario, serum samples were sent to our laboratory accompanied by the NT measurement, enabling the calculation of a combined risk at our laboratory. Alternatively, the NT measurements were sent to our laboratory after reporting the risk based on the biochemical parameters, for subsequent calculation and reporting of a combined risk. The results were sent to the centres by fax or e-mail and usually also by conventional mail. In the present study, only the NT-combined risk calculations as calculated at our laboratory were included in the epidemiological analysis.

Women were requested to fill in a short questionnaire about the pregnancy outcome. If necessary, a second request to report the outcome of the pregnancy was done by mail. This procedure was in agreement with Dutch and European legislation on the protection of privacy. Exclusion criteria for the epidemiological analysis were (besides non-combined risk calculation) multiple pregnancies, IDDM pregnancies, pregnancies in which CRL values were either smaller than $38 \mathrm{~mm}$ or larger than $84 \mathrm{~mm}$ and prolonged GA.

\section{NT measurement}

Centres were informed that it was expected that the NT measurements were carried out according to the FMF protocol and that the sonographers involved were FMF-certified (www.fetalmedicine. $\mathrm{nl}$ ). Whether these conditions were actually met was checked against the register on the FMF website (25 March 2005).

\section{Risk estimation}

Assay values and NT measurements were transformed into a risk for Down syndrome at term with the software package 1T-risks (version 1.7, 1999, PerkinElmer- Wallac, Turku, Finland). This software package uses the basic principles of risk calculation as published previously (Reynolds, 1994). Briefly, from PAPP-A and fB-hCG assay values and from NT and CRL values (in $\mathrm{mm}$ ) of NT measurements, the MoM values were calculated. Log transformations of these MoM values were compared to Gaussian distributions of the normal and Down syndrome pregnancies (Nicolaides et al., 1998; Cuckle and van Lith, 1999) to obtain likelihood ratios. From these ratios and the maternal agespecific risk (Cuckle et al., 1987), the Down syndrome risks were calculated. For the biochemical parameters, median concentrations were statistically modelled using data from our own laboratory. The median 
Prenat Diagn 2006; 26: 711-718.

NT were those from Nicolaides et al., (1998). PAPP-A and f $\beta$ hCG MoMs were corrected for maternal weight by reciprocal-linear regression. No corrections were made with respect to smoking behaviour and IDDM-status. A risk of 1 in 250 or higher at term was considered an increased risk for a Down syndrome pregnancy (screen positive).

\section{Quality assurance}

The quality of the entire risk calculation process was assessed daily and monthly by participating in both internal and external (UK-NEQAS, Edinburgh, UK) quality control programmes for PAPP-A, $\mathrm{f} \beta$-hCG, and the risk calculation. 1T-Risks software was the risk calculation software during the study period. The current risk calculation software of our laboratory is the more sophisticated Lifecycle Elipse (PerkinElmer, Turku, Finland). A subset of the results of the 1T-risks programme was re-calculated in Lifecycle Elipse to validate the original 1T-risks results. The calculation of the expected number of Down syndrome cases, the expected detection rate (DR), and the false-positive rate (FPR) for the population under investigation was done with the software package DSQA tools, version 1.0.0. (Media Innovations, Leeds, UK).

\section{RESULTS}

Between July 2002 and May 2004 the RIVM received 7766 requests for a first-trimester test. For various reasons, as shown in Figure 1, 105 requests were excluded from this study. From the remaining requests, 2827 were excluded because the combined risk calculation was done in one of the centres and not in our own laboratory. From the remaining requests, 4033 cases were ultimately available for epidemiological analysis. The median maternal age of all first-trimester requests was 36.6 (range 18-47) years, that of those excluded from the analysis was 36.7 and of those included in the analysis was 36.5, the age distributions thus being alike. The GA of all first-trimester requests at blood sampling was 11.5 (range 8.013.6) weeks. Closer examination showed that the distribution of GA was biphasic with medians of 10.3 and 12.1 weeks, respectively. This distribution coincided with the distribution of the GA of requests included in the epidemiological analysis (median GA: 12.1 weeks) versus those excluded (GA: 10.5 weeks).

The estimated risk for Down syndrome in the analysed group on the basis of maternal age, fetal NT, and maternal serum PAPP-A and $\mathrm{f} \beta$-hCG was 1 in 250 or higher in $4.7 \%$ of the normal pregnancies and in $71 \%(15 / 21)$ of those with Down syndrome. The odds of being affected given a positive result (OAPR) was 1 in 14 . The median MoM values of PAPP-A and $\mathrm{f} \beta$-hCG, that were not corrected for weight, were $0.94 \pm 0.02$ and $0.98 \pm 0.03$, respectively. Within the analysed group, maternal weight of $96.2 \%$ cases was known, with a median of $67 \mathrm{~kg}$. 
Prenat Diagn 2006; 26: 711-718.

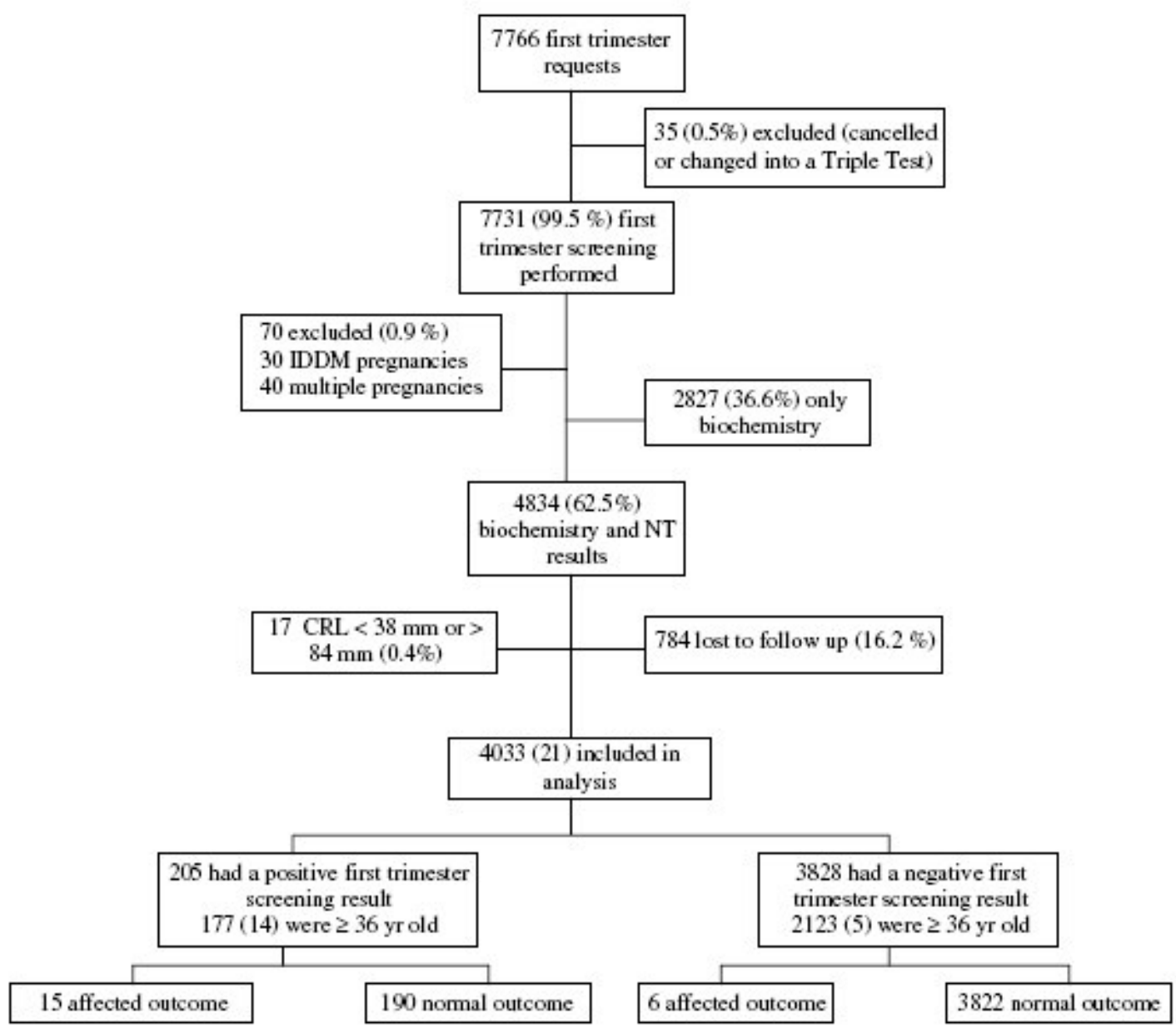

Figure 1-Flow diagram showing the fate of requests in this study. Numbers in parentheses are the numbers of pregnancies with a foetus with Down syndrome 
Prenat Diagn 2006; 26: 711-718.

Table 1-Screening characteristics of the Down syndrome cases

\begin{tabular}{|c|c|c|c|c|c|}
\hline Case & $\begin{array}{c}\text { Maternal } \\
\text { age at exp. } \\
\text { day } \\
\text { delivery }\end{array}$ & MoM PAPP-A & MoM $\mathrm{f} \beta-\mathrm{HCG}$ & $\begin{array}{c}\text { MoM } \\
\text { NT }\end{array}$ & $\begin{array}{c}\text { Risk a } \\
\text { term }\end{array}$ \\
\hline \multicolumn{6}{|c|}{ Detected } \\
\hline 1 & 43 & 0.11 & 1.10 & 1.83 & 10 \\
\hline 2 & 39 & 0.31 & 1.19 & 1.90 & 10 \\
\hline 3 & 38 & 0.26 & 0.87 & 1.90 & 10 \\
\hline 4 & 41 & 0.17 & 5.14 & 1.25 & 10 \\
\hline 5 & 37 & 0.22 & 3.01 & 1.58 & 10 \\
\hline 6 & 29 & 0.88 & 2.50 & 4.29 & 10 \\
\hline 7 & 38 & 0.50 & 2.87 & 2.13 & 10 \\
\hline 8 & 38 & 0.51 & 2.19 & 2.97 & 10 \\
\hline 9 & 37 & 0.46 & 1.84 & 2.61 & 10 \\
\hline 10 & 42 & 0.19 & 2.19 & 1.12 & 20 \\
\hline 11 & 39 & 0.52 & 0.46 & 2.29 & 20 \\
\hline 12 & 40 & 0.30 & 1.97 & 1.31 & 40 \\
\hline 13 & 37 & 1.85 & 3.31 & 1.52 & 90 \\
\hline 14 & 40 & 0.97 & 3.04 & 1.35 & 110 \\
\hline 15 & 38 & 0.46 & 3.12 & 0.62 & 160 \\
\hline \multicolumn{2}{|c|}{$\begin{array}{l}\text { Median MoM } \\
\text { Non-detected }\end{array}$} & 0.46 & 2.19 & 1.83 & \\
\hline 16 & 44 & 1.04 & 0.75 & 1.34 & 300 \\
\hline 17 & 36 & 0.18 & 0.98 & 0.87 & 430 \\
\hline 18 & 34 & 0.14 & 1.17 & 0.89 & 470 \\
\hline 19 & 39 & 0.54 & 0.77 & 1.04 & 1300 \\
\hline 20 & 37 & 0.88 & 1.19 & 0.76 & 3100 \\
\hline 21 & 42 & 1.89 & 1.29 & 0.87 & 6300 \\
\hline \multicolumn{2}{|c|}{ Median MoM } & 0.71 & 1.08 & 0.88 & \\
\hline
\end{tabular}

The median weight-corrected MoM values ( $\pm 95 \% \mathrm{CI}$ ) of PAPP-A and $\mathrm{f} \beta$-hCG were $1.06 \pm$ 0.02 and $1.07 \pm 0.03$, respectively. The median MoM value ( $\pm 95 \%$ CI) of NT was $0.83 \pm$ 0.01 . A summary of the screening characteristics of the Down syndrome cases is presented in Table 1. Six Down syndrome foetuses in this study were not identified by the screening test. Notably, the median NT MoM of the non-detected cases was 0.88 . Of the 17 foetuses for which the age at term could be reconstructed, all but two were born (well) below a GA of 19 weeks, two were born below a GA of 23 weeks and two were born at 38-39 weeks.

Within the screened population, three cases of trisomy 18 , six of trisomy 13 and two of triploidy were found. The screening characteristics of those cases are summarized in Table 2. To specify the differences between centres, the NT measurement (the only factor in the risk calculation that is centre-specific) was further investigated. Because the NT data were not recorded at the level of the individual operator, but rather at the centre-level, the performance of operators was indirectly assessed by evaluating the performance of each centre. Results of this analysis are presented in Figure 2. They show that the NT participating centres with 60 NT measurements or more during the study period. Lines are fitted by least squares weighted natural logarithmic regression analysis through the raw NT/CRL measurements of individual centres. Solid lines: FMF-certified centres; Dashed lines: non-FMF certified centres; Squared dots: FMF curve (Pandya et al., 1995; Snijders et al., $1998,1999)$ measurements of many centres are below or far below the FMF curve. Centres 
with an FMF license does not necessarily appear to perform better than centres without this license. The median NT MoM of the six centres that performed more than 60 NT measurements during the study period and without a license was $0.81 \mathrm{MoM}$ (range $0.53-$ $1.00 ; n=1986$ ), while that of the 11 licensed centres was 0.84 (range $0.66-0.99 ; n=$ 2555). The median of all the NT MoM of centres (certified and noncertified) that performed less than 60 NT measurements during the study period was 0.83 (range $0.76-1.13 ; n=$ 284).

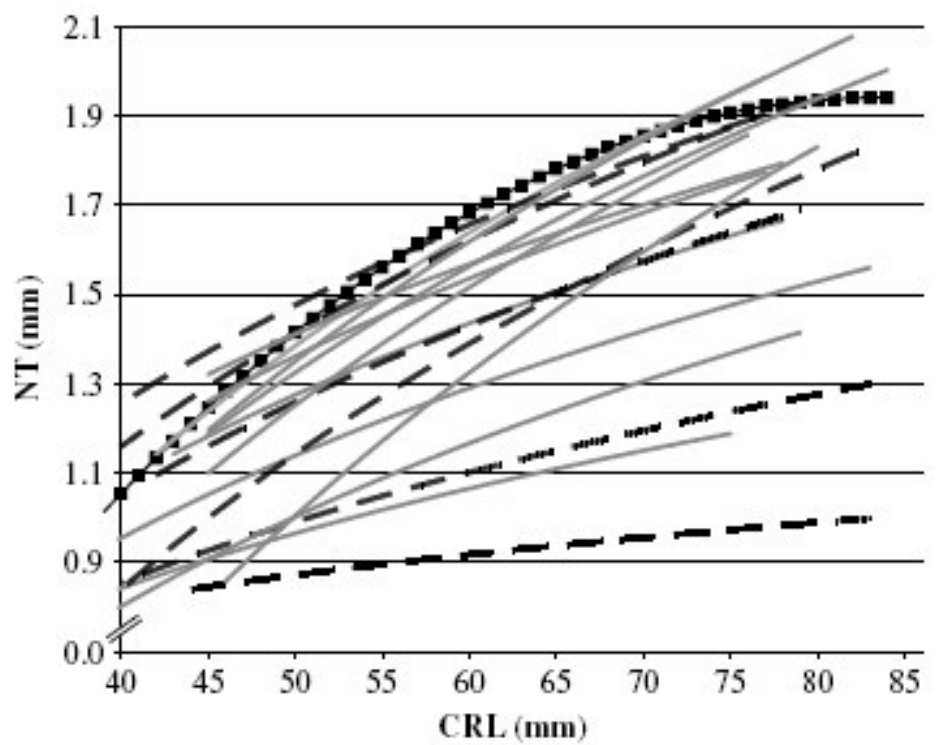

Figure 2-Nuchal translucency measurement related to crown-rump length of 17

Table 2-Screening characteristics of the trisomy 18 and 13

\begin{tabular}{|c|c|c|c|c|}
\hline Case & $\begin{array}{l}\text { Maternal } \\
\text { age }\end{array}$ & $\begin{array}{c}\text { MoM } \\
\text { PAPP-A }\end{array}$ & $\begin{array}{c}\text { MoM } \\
\mathrm{f} \beta \text {-hCG }\end{array}$ & $\begin{array}{c}\text { MoM } \\
\text { NT }\end{array}$ \\
\hline \multicolumn{5}{|c|}{ Trisomy 18} \\
\hline 1 & 41 & 0.15 & 0.67 & 2.15 \\
\hline 2 & 36 & 0.17 & 0.14 & 1.27 \\
\hline 3 & 33 & 0.39 & 0.10 & 0.65 \\
\hline \multicolumn{5}{|c|}{ Trisomy 13} \\
\hline 1 & 37 & 0.08 & 0.34 & 2.15 \\
\hline 2 & 37 & 0.09 & 1.92 & 1.16 \\
\hline 3 & 38 & 0.14 & 0.69 & 1.07 \\
\hline 4 & 37 & 0.25 & 0.15 & 1.53 \\
\hline 5 & 36 & 0.09 & 0.28 & 0.68 \\
\hline 6 & 32 & 0.33 & 0.79 & 0.90 \\
\hline \multicolumn{5}{|c|}{ Triploidy } \\
\hline 1 & 41 & 0.07 & 0.40 & 0.49 \\
\hline 2 & 36 & 0.18 & 0.11 & 0.87 \\
\hline
\end{tabular}


Prenat Diagn 2006; 26: 711-718.

\section{DISCUSSION}

This is the first report on the results of first-trimester combined screening test in the Netherlands in clinical practice. The aim of this study was to evaluate the strengths and weaknesses of the first-trimester screening test in a setting with many participating centres. In the study period, 7731 first-trimester combined tests were performed and 4033 of these were included in the analysis. We identified 15 of the 21 foetuses with Down syndrome $(71 \%)$ with a combination of serum markers, nuchal translucency thickness, and maternal age, with an FPR of $4.7 \%$.

In general, the Dutch population of pregnant women is among the oldest group of pregnant women in Europe (Eurostat, 2005). Because of this, and probably owing to the earlier screening policy of the government, relatively old pregnant women opt for Down syndrome screening. The maternal age distribution as described in this study is therefore not surprising and comparable to the age distribution of the triple test conducted over the years in our laboratory.

On the basis of the maternal age distribution, 15 pregnancies with Down syndrome at term can be expected. The higher number of 21 affected pregnancies as described in this study may partly be explained by the fact that approximately $30 \%$ of Down syndrome pregnancies end in a miscarriage between the first trimester and term (Morris et al., 1999; Snijders et al., 1999). The high rate of reported Down syndrome pregnancies over the expected rates gives some confidence that all the Down syndrome cases in the study were actually reported. At least 15 of the 21 Down syndrome pregnancies in this study were indeed detected before 23 weeks of pregnancy. Taking into account the fetal loss until term, the estimated DR of this study is 73\% (CI: 45-92\%). Although the number of Down syndrome cases in this study is low (and thus the CI rather wide), there are still some points to be made concerning the performance characteristics of the current programme.

It cannot be excluded that, on the basis of an exceptionally disadvantageous biochemical risk that was reported, a number of pregnant women decided not to wait for an NT measurement but to have a diagnostic invasive test immediately. Moreover, pregnant women may have decided not to do an NT measurement because their risk on the basis of biochemical analysis alone was very low. In both instances, Down syndrome cases born from these pregnancies would have to be found in the group of 2827 , for which only the biochemistry results are known (Figure 1 ). Indeed, there were four reported Down syndrome cases in this group, with risks of 1 in 30,1 in 40,1 in 130, and 1 in 490, respectively, on the basis of biochemistry only. Theoretically, if for these Down Syndrome cases the NT measurement was known and the risk would have been over or equal to 1 in 250, the DR in this study would have been $76 \%$ (19 detected cases out of 25). As we state very clearly to requestors that biochemical results should always be combined with the result of an NT measurement to obtain a high quality risk assessment, and since we have no indication that the requestors did otherwise, the DR of $71 \%$ is probably accurate.

Several other studies have showed results comparable to the modelled expectations of Cuckle and Arbuzova, 2004 (e.g. Bindra et al., 2002; Nicolaides et al., 2005 Schuchter et al., 2002; Spencer et al., 2000a; Stenhouse et al., 2004; von Kaisenberg et al., 2002), while others obtained results similar to this study (e.g. Muller et al., 2003). A higher DR thus seems feasible. The NT measurement, when executed properly, is a reliable parameter for Down syndrome screening. It is also vulnerable and therefore its quality should be monitored (Malone and D'Alton, 2003). 
Prenat Diagn 2006; 26: 711-718.

The centres measured smaller NT than the FMF did (see Figure 2), while the median MoM PAPP-A and median MoM $\mathrm{f} \beta$-hCG values were rather normal. The data in Table 1 illustrate that NT measurements of the missed Down syndrome cases invariably were low. Thus, it may be concluded that the low NT measurements of this study contributed importantly to the relatively low DR (71\%, where $84 \%$ was expected) and low FPR $(4.7 \%$, where $7.4 \%$ was expected). Previously, it was suggested and demonstrated that the use of centreor operatorspecific medians was beneficial to the performance of a screening programme (Logghe et al., 2003; Wald et al., 2003). In the current study, the number of NT measurements per centre was too low to recalculate the risks using centre-specific medians. To demonstrate the effect of centre-specific NT-medians, all available NT measurements were used to model a new NT median curve. Recalculating all the risks using this curve produced a DR of $76 \%$ and an FPR of $7.0 \%$, which is more in line with the expected performance characteristics associated with the age distribution of the population under investigation.

In a survey of 13 Dutch centres, van den Berg et al. (2005a) also concluded that NT measurements in the Netherlands did not reach the quality level that is required for reliable risk estimation. Among the shortcomings were the magnitude of the image, the placing of the callipers, and the visibility of the difference between the amnion and fetal skin. Placing the callipers correctly is considered as one of the most important aspects of the NT measurement. A misplacement of even a part of a millimetre will have a substantial effect on the estimation of the risk for Down syndrome (Herman et al., 2000).

Improving the NT measurement by closely following the FMF guidelines may increase the DR for Down syndrome, even by as much as 30 to 84\% (Monni et al., 1997; Wapner et al., 2003). In the Netherlands there is a growing awareness about the importance of measuring NT accurately and the importance of reliable median NT curves. Thus, the medical centre of the Free University of Amsterdam has set up training and peer groups for NT measurement and, in the meanwhile, has adopted the practice of operatorspecific medians. The Dutch Society for Obstetrics and Gynaecology has issued standards for quality assurance of the NT measurement. The effects of these measures on the performance of the programme will be the object of future study.

Two recent studies that adopted the FMF protocol published the median NT as significantly lower than the FMF medians (Crossley et al., 2002; Stenhouse et al., 2004). In this regard, it is of interest that the quality of the sonographic equipment used appears to affect the NT measurement (Wald et al., 2003). As the performance of the equipment has changed over the years, so also may have the median values of the NT in healthy foetuses. Further study should address the question of whether training and experience alone or other factors also underlie the tendency to measure lower NT in the unaffected population.

While NT measurement seems to be the major means for improvement in the performance of the programme at present, significant improvements may also be expected from a better setting of the medians for the biochemical parameters. Besides, standardisation of the determination of GA during blood sampling may improve the performance of the screening programme. In the current study, the requesting medical practitioners' statement on the GA during blood sampling was leading. It is known that individual requestors in the Netherlands may determine the GA by the LMP, a dating scan, or a mixture of the two (e.g. LMP, unless the dating scan differs more than 7 days - in which case the dating becomes leading).

Determining the GA exclusively on a dating scan may lead to some improvement (Bishop et al., 1997). The parameters of the serum test may also be useful to detect trisomy 13 and trisomy 18. A low MoM PAPP-A and MoM f $\beta$-hCG $(<0.25)$ seem to point at a trisomy 18 or a trisomy 13 pregnancy, as both aneuploidies are associated with decreased maternal serum 
PAPP-A and $\mathrm{f} \beta$-hCG (Tul et al., 1999; Spencer et al., 2000b). Furthermore, the serum test may be useful to detect digynic triploidy, as this genetic abnormality is associated with markedly decreased maternal serum $\mathrm{f} \beta$-hCG and PAPP-A (Yaron et al., 2004). With the introduction of new software, trisomy 18 and 13 have become detectable. Whether or not this will be included in the Dutch screening policy is a matter of debate (see below).

The centralised first-trimester screening programme at the RIVM, as described here, is only one of many possible settings. An alternative setting is the one stop clinic for the assessment of risk for foetal abnormality (OSCAR) where biochemical testing, ultrasound examination, and counselling are carried out in a 1-h visit. OSCAR is typically done in small centres, with an average of less than a thousand tests per annum. When adopted as a national screening policy this may result in a multitude of screening policies, software settings, and levels of expertise. The responsibility for the collection of follow-up data may be felt quite differently among different OSCAR centres, the staff of such small centres probably being too small to be fully dedicated to quality assurance issues. From a standpoint of quality assurance, the OSCAR setting is therefore almost unmanageable. This weakness is considered far more important than the strengths of the OSCAR setting, e.g. the swiftness of producing a result and probably the easier accessibility for pregnant women because of the availability of many local centres. The strengths of centralised screening include an accurate quality control and validation, and maintenance of the software, by dedicated staff. A potential weakness may occur in the logistics of results and samples. As is the case of studies in a routine clinical setting in general, this study suffered from the lack of completeness of data. Especially, the nonreporting of locally calculated combined risks to our laboratory for evaluation purposes and the incomplete follow up of the outcomes of pregnancies (Figure 1 ) resulted in a considerable loss of requests for the epidemiological analysis. Whether the inability to obtain an adequate image (NT failure rate) contributed considerably to the number of excluded tests is not known. Therefore, the important criteria for a centralised programme are validation and maintenance of the software, optimal communication, a central project leader, and optimal logistics for information exchange between laboratories and screening centres.

In its report, the Dutch Health Council concluded that the first-trimester combined test was the screening method of choice for Down syndrome in routine practice (Health Council of the Netherlands, 2004), and is to be preferred over the triple test. Its conclusion was mainly based on a meta-analysis of Cuckle and Arbuzova (2004), who reported a sensitivity of $82 \%$ for a 5\% FPR for the combined test. In the current study, it could not confidently be established that a comparably high DR was reached. The odds of being affected given a positive result (OAPR) of 1 in 14, however, is superior to that of the OAPR of 1 in 34 of the triple test as established in a comparable population (our own, unpublished data on 5491 triple tests performed by our laboratory between 1999 and 2001). For this reason, in this multi-centre setting the combined test is more promising and should be preferred over the triple test.

The Dutch governmental policy for prenatal screening has changed recently. All pregnant women are now allowed to be informed on the risk estimation tests for Down syndrome by gynaecologists and midwives, thus offering them the possibility of informed choice for the first time (van den Berg et al., 2005b). This new policy resulted in a growing number of requests for the first-trimester combined test (Schielen et al., 2005) and an increasing familiarity with this test among pregnant women.

As an effectuation of this new policy, governmental guidelines have been issued for a formal centralized screening programme for Down syndrome, which is to be fully active by early 2007. The informal firsttrimester test screening programme of the RIVM, with one central 
Prenat Diagn 2006; 26: 711-718.

laboratory for biochemical assay and risk calculation and a restricted number of liased centres where requests are registered and NT measurements performed, as described in this study, may be seen as a successful pilot of this governmental programme, and an important learning experience.

\section{ACKNOWLEDGEMENTS}

The authors are indebted to Prof. Dr H. Cuckle for critically reading the manuscript.

\section{REFERENCES}

Berg M van den, Kleinveld JH, Sander MJ, Van Vugt JMG, Timmermans DRM. 2005a. Quality of nuchal translucency measurements: an exploratory study into their performance and evaluation. Ned Tijdschr Geneeskd 30: 16911696, (article in Dutch; English abstract available).

Berg M van den, Timmermans DRM, Kleinveld JH, Garcia E, Van Vugt JMG, Van der Wal G. 2005b. Accepting or declining the offer of prenatal screening for congenital defects: test uptake and women's reasons. Prenat Diagn $\mathbf{2 5}$ : 84-90.

Bindra R, Heath V, Liao A, Spencer K, Nicolaides KH. 2002. Onestop clinic for assessment of risk for trisomy 21 at 11-14 weeks: a prospective study of 15030 pregnancies. Ultrasound Obstet Gynecol 20: 219-225.

Bishop JC, Dunstan FD, Nix AB. 1997. The effects of gestation dating on the calculation of patient-specific risks in down's syndrome screening: multivariate case. Ann Clin Biochem 34: 55-60.

Crossley JA, Aitken DA, Cameron AD, McBride E, Connor JM. 2002. Combined ultrasound and biochemical screening for down's syndrome in the first trimester: a Scottish multicentre study. Br J Obstet Gynaecol 109: 667-676.

Cuckle HS, van Lith JM. 1999. Appropriate biochemical parameters in first-trimester screening for down syndrome. Prenat Diagn 19: 505-512.

Cuckle HS, Arbuzova S. 2004. Multimarker serum screening for chromosomal abnormalities. In Genetic Disorders and the Fetus; Diagnosis, Prevention, and Treatment, Milunsky A (ed.). Johns Hopkins University Press: Baltimore; 795-835.

Cuckle HS, Wald NJ, Thompson SG. 1987. Estimating a woman's risk of having a pregnancy associated with down's syndrome using her age and serum alpha-fetoprotein level. Br J Obstet Gynaecol 94: 387-402.

Eurostat, Statistical Office of the European Communities. Population and social conditions, mean age of women at child bearing. 2002- data. http://epp.eurostat.cec.eu.int. Retrieved October 12, 2005.

Health Council of the Netherlands. Prenatal screening; Down syndrome, neural tube defects, routine ultrasound. Health Council of the Netherlands: Hague; 2001. publication no. 2001/11 (In Dutch).

Health Council of the Netherlands. Prenatal Screening (2); Down's syndrome, Neural Tube Defects. Health Council of the Netherlands: Hague; 2004. publication no. 2004/06 (In Dutch).

Herman A, Dreazen E, Samandarov A, Bukovsky Y, Weinraub Z, Maymon R. 2000. On-to-on versus on-to-out nuchal translucency measurements. Ultrasound Obstet Gynecol 15: 126-130.

Kaisenberg CS von, Gasiorek-Wiens A, Bielicki M, et al. German Speaking Down Syndrome Screening Group. 2002. Screening for trisomy 21 by maternal age, fetal nuchal translucency

and maternal serum biochemistry at 11-14 weeks: a German multicenter study. J Matern Fetal Neonatal Med 12: 89-94.

Logghe H, Cuckle H, Sehmi I. 2003. Centre-specific ultrasound nuchal translucency medians needed for down's syndrome screening. Prenat Diagn 23: 389-392.

Malone FD, D'Alton ME. Society for Maternal-Fetal Medicine 2003. First-trimester sonographic screening for down syndrome. Obstet Gynecol 1025: 1066-1079.

Monni G, Zoppi MA, Ibba RM, Floris M. 1997. Fetal nuchal translucency test for down's syndrome. Lancet 350: 1631. 
Prenat Diagn 2006; 26: 711-718.

Morris JK, Wald NJ, Watt HC. 1999. Fetal loss in down syndrome pregnancies. Prenat Diagn 19: 142-145.

Muller F, Benattar C, Audibert F, Roussel N, Dreux S, Cuckle H. 2003. First-trimester screening for down syndrome in France combining fetal nuchal translucency measurement and biochemical markers. Prenat Diagn 23: 833-836.

Nicolaides KH, Snijders RJM, Cuckle HS. 1998. Correct estimation of parameters for ultrasound nuchal translucency screening. Prenat Diagn 18: 511-523.

Nicolaides KH, Spencer K, Avgidou K, Faiola S, Falcon O. 2005. Multicenter study of first-trimester screening for trisomy 21 in 75821 pregnancies: results and estimation of the potential impact of individual risk-orientated twostage first-trimester screening. Ultrasound Obstet Gynecol 25: 221-226.

Pandya PP, Snijders RJ, Johnson SP, De Lourdes Brizot M, Nicolaides KH. 1995. Screening for fetal trisomies by maternal age and fetal nuchal translucency thickness at 10 to 14 weeks of gestation. $\mathrm{Br} \mathrm{J}$ Obstet Gynaecol 102(12): 957-962.

Reynolds TM. 1994. Screening by test combination: a statistical overview. In Screening for Down's syndrome, Grudzinskas JG, Chard T, Chapman MG, Cuckle H (eds). Cambridge University Press: New York; 47-71.

Royston P, Thompson SG. 1992. Model-based screening by risk with application to down's syndrome. Stat Med 11: 257-268.

Schielen PCJI, Elvers LH, Loeber JG. Towards a national programme for down syndrome and neural tube defect screening in the Netherlands. Gynaecology, Obstetrics, and Reproductive Medicine in Daily Practice Proceedings of the 15th Congress of Gynaecology, Elsevier, Rotterdam, The Netherlands, May 2005.

Schuchter K, Hafner E, Stangl G, Metzenbauer M, Hofinger D, Philipp K. 2002. The first trimester 'combined test' for the detection of down syndrome pregnancies in 4939 unselected pregnancies. Prenat Diagn 22: 211-215.

Snijders RJ, Noble P, Sebire N, Souka A, Nicolaides KH. 1998. UK multicentre project on assessment of risk of trisomy 21 by maternal age and fetal nuchal-translucency thickness at 10-14 weeks of gestation. Fetal medicine foundation first trimester screening group. Lancet 352: 343-346.

Snijders RJ, Sundberg K, Holzgreve W, Henry G, Nicolaides KH. 1999. Maternal age- and gestation-specific risk for trisomy 21. Ultrasound Obstet Gynecol 13: 167-170.

Spencer K, Souter V, Tul N, Snijders R, Nicolaides KH. 1999. A screening programme for trisomy 21 at 10-14 weeks using fetal nuchal translucency, maternal serum free beta-human chorionic gonadotropin and pregnancyassociated plasma protein-A. Ultrasound Obstet Gynecol 13: 231-237.

Spencer K, Spencer CE, Power M, Moakes A, Nicolaides KH. 2000a. One stop clinic for assessment of risk for fetal anomalies: a report of the first year of prospective screening for chromosomal anomalies in the first trimester. $\mathrm{Br} \mathrm{J}$ Obstet Gynaecol 107: 1271-1275.

Spencer K, Ong C, Skentou H, Liao AWH, Nicolaides K. 2000b. Screening for trisomy 13 by fetal nuchal translucency and maternal serum free beta-hCG and PAPP-A at 10-14 weeks of gestation. Prenat Diagn 20: 411-416.

Spencer K, Spencer CE, Power M, Dawson C, Nicolaides KH. 2003. Screening for chromosomal abnormalities in the first trimester using ultrasound and maternal serum biochemistry in a one-stop clinic: a review of three years prospective experience. Br J Obstet Gynaecol 110(3): 281-286.

Stenhouse EJ, Crossley JA, Aitken DA, Brogan K, Cameron AD, Connor JM. 2004. First-trimester combined ultrasound and biochemical screening for Down syndrome in routine clinical practice. Prenat Diagn 24: 774-780.

Tul N, Spencer K, Noble P, Chan C, Nicolaides K. 1999. Screening for trisomy 18 by fetal nuchal translucency and maternal serum free beta-hCG and PAPP-A at 10-14 weeks of gestation. Prenat Diagn 19: 1035-1042.

Wald NJ, Hackshaw AK. 1997. Combining ultrasound and biochemistry in first-trimester screening for down's syndrome. Prenat Diagn 17: 821-829.

Wald NJ, Rodeck C, Hackshaw AK, Walters J, Chitty L, Mackinson AM. SURUSS Research Group. 2003. First and second trimester antenatal screening for down's syndrome: the results of the serum, urine and ultrasound screening study (SURUSS). Health Technol Assess 7: 1-77. 
Prenat Diagn 2006; 26: 711-718.

Wapner R, Thom E, Simpson JL, et al. 2003. First trimester maternal serum biochemistry and fetal nuchal translucency screening (BUN) study group. First-trimester screening for trisomies 21 and 18. Firsttrimester screening for trisomies 21 and 18. $N$ Engl J Med 349:

1405-1413.

Yaron Y, Ochshorn Y, Tsabari S, Shira AB. 2004. First-trimester nuchal translucency and maternal serum free betahCG and PAPP-A can detect triploidy and determine the parental origin. Prenat Diagn 24: 445-450. 